\title{
Determinations of flavor ratios and flavor transitions of astrophysical neutrinos
}

\section{Guey-Lin Lin ${ }^{* \dagger}$}

Institute of Physics, National Chiao-Tung University, Hsinchu 300, Taiwan

E-mail: glinemail.nctu.edu.tw

\section{T. C. Liu}

Leung Center for Cosmology and Particle Astrophysics, National Taiwan University, Taipei 106, Taiwan

E-mail: diewanger@gmail.com

\section{Kwang-Chang Lai}

Physics Group, Center for General Education, Chang Gung University, Kwei-Shan 333, Taiwan E-mail: kclai@mail.nctu.edu.tw

\section{A. Huang}

Department of Energy and Resources, National United University, Miao-Li 36003, Taiwan

E-mail: mahuang@nuu.edu.tw

\begin{abstract}
We argue that an effective flavor discrimination in neutrino telescopes is the key to probe the flavor ratios of astrophysical neutrinos at the source and flavor transition mechanisms of these neutrinos during their propagations from the source to the Earth. We first discuss how well one can reconstruct the flavor ratios of astrophysical neutrinos at the source. We then discuss how to probe flavor transition mechanisms of propagating astrophysical neutrinos. In this regard, we propose a model independent parametrization for neutrino flavor transitions, with the neutrino oscillation as a special case.
\end{abstract}

35th International Conference of High Energy Physics - ICHEP2010,

July 22-28, 2010

Paris France

\footnotetext{
*Speaker.

${ }^{\dagger}$ Supported by the National Science Council of Taiwan under Grant No. 96-2112-M-009-023-MY3
} 


\section{Determining Flavor Ratios of Astrophysical Neutrinos}

It has been demonstrated that [1] the event ratio of muon tracks to showers can be measured to a $10 \%$ accuracy for a decade of data taking in IceCube with a neutrino flux in the order of WaxmanBahcall bound [2]. The above accuracy in measuring track to shower ratio can be translated into a comparable accuracy for determining the flux ratio $R \equiv \phi\left(v_{\mu}\right) /\left(\phi\left(v_{e}\right)+\phi\left(v_{\tau}\right)\right)$. We have pointed out that a well determined $R$ is still not sufficient for constraining the neutrino flavor ratio at the source, which is characterized by the vector $\Phi_{0}=\left(\phi_{0}\left(v_{e}\right), \phi_{0}\left(v_{\mu}\right), \phi_{0}\left(v_{\tau}\right)\right)^{T}$ with [3]

$$
\begin{aligned}
& \phi_{0}\left(v_{e}\right)+\phi_{0}\left(v_{\mu}\right)+\phi_{0}\left(v_{\tau}\right)=1, \\
& \phi_{0}\left(v_{\alpha}\right) \geq 0, \text { for } \alpha=e, \mu, \tau,
\end{aligned}
$$

where each $\phi_{0}\left(v_{\alpha}\right)$ is the sum of neutrino and antineutrino fluxes. On the other hand, we have shown that [4] it is possible to discriminate between an astrophysical pion source with $\Phi_{0}=$ $(1 / 3,1 / 2,0)^{T}$ and a muon damped source with $\Phi_{0}=(0,1,0)^{T}$ [5] at the $3 \sigma$ level, provided that the separation between the tau shower and the electron shower, namely the measurement of the parameter $S \equiv \phi\left(v_{e}\right) / \phi\left(v_{\tau}\right)$, can be done as effectively as $R$ in neutrino telescopes. To reach this conclusion, we have taken into account the following uncertainties ( $1 \sigma$ ranges) of neutrino mixing parameters [6]

$$
\sin ^{2} \theta_{12}=0.32_{-0.02}^{+0.02}, \sin ^{2} \theta_{23}=0.45_{-0.06}^{+0.09}, \sin ^{2} \theta_{13}<0.019 \text {. }
$$

We conclude that tau neutrino identification is crucial for developing the neutrino flavor astronomy.

We also consider the detection of very high energy astrophysical neutrinos, i.e., $E_{v}>33 \mathrm{PeV}$. In such an energy range, the tau lepton originated from the tau neutrino behaves more like a track rather than a shower while the electron neutrino only give rises to a shower signature. Therefore the more appropriate flux ratio parameters in such a case are $R^{\prime} \equiv \phi\left(v_{e}\right) /\left(\phi\left(v_{\mu}\right)+\phi\left(v_{\tau}\right)\right)$ and $S^{\prime} \equiv$ $\phi\left(v_{\mu}\right) / \phi\left(v_{\tau}\right)$. We found that $R^{\prime}$ is a more sensitive parameter than $R$ for reconstructing the neutrino flavor ratio at the source, if both parameters are measured to the same accuracy [7]. Furthermore, it is interesting to note that a further measurement on $S^{\prime}$ does not improve the reconstruction of initial neutrino flavor ratios. In fact, due to the approximate $v_{\mu}-v_{\tau}$ symmetry [8,9], $S^{\prime}$ is always close to 1 irrespective of the initial neutrino flavor ratio. We note that $R^{\prime}$ can be measured in the radio extension of IceCube detector [10], which aims for detecting very high energy neutrinos such as those produced by the interactions between ultrahigh energy cosmic rays and cosmic microwave background radiations.

\section{Probing Flavor Transitions of Astrophysical Neutrinos}

The effect of neutrino flavor transition processes occurring between the astrophysical source and the Earth is represented by the matrix $P$ such that

$$
\Phi=P \Phi_{0}
$$

where $\Phi=\left(\phi\left(v_{e}\right), \phi\left(v_{\mu}\right), \phi\left(v_{\tau}\right)\right)^{T}$ is the flux of neutrinos reaching to the Earth. We note that our convention implies $P_{\alpha \beta} \equiv P\left(v_{\beta} \rightarrow v_{\alpha}\right)$. The matrix $P$ can be easily calculated for standard 
neutrino oscillation model. However we should keep $P$ as general as possible for accommodating other flavor transition models. It is convenient to parametrize the initial flux of neutrinos by [4]

$$
\Phi_{0}=\frac{1}{3} \mathbf{V}_{1}+a \mathbf{V}_{2}+b \mathbf{V}_{3}
$$

where $\mathbf{V}_{1}=(1,1,1)^{T}, \mathbf{V}_{2}=(0,-1,1)^{T}$, and $\mathbf{V}_{3}=(2,-1,-1)^{T}$. The ranges for $a$ and $b$ are $-1 / 3+$ $b \leq a \leq 1 / 3-b$ and $-1 / 6 \leq b \leq 1 / 3$. Likewise, one can also write the flux on Earth in the same basis

$$
\Phi=\kappa \mathbf{V}_{1}+\rho \mathbf{V}_{2}+\lambda \mathbf{V}_{3}
$$

It is easy to show that

$$
\left(\begin{array}{c}
\kappa \\
\rho \\
\lambda
\end{array}\right)=\left(\begin{array}{lll}
Q_{11} & Q_{12} & Q_{13} \\
Q_{21} & Q_{22} & Q_{23} \\
Q_{31} & Q_{32} & Q_{33}
\end{array}\right)\left(\begin{array}{c}
1 / 3 \\
a \\
b
\end{array}\right)
$$

where $Q=\mathbf{A}^{-1} P \mathbf{A}$ with

$$
\mathbf{A}=\left(\begin{array}{ccc}
1 & 0 & 2 \\
1 & -1 & -1 \\
1 & 1 & -1
\end{array}\right)
$$

In other words, $Q$ is related to $P$ by a similarity transformation where columns of the transformation matrix $\mathbf{A}$ correspond to vectors $\mathbf{V}_{1}, \mathbf{V}_{2}$, and $\mathbf{V}_{3}$, respectively.

For the flux-conserving case during the neutrino propagations, one has $\kappa=1 / 3$ which requires $Q_{11}=1$ and $Q_{12}=Q_{13}=0$, since the coefficients $a$ and $b$ are arbitrary. Furthermore, in the exact $v_{\mu}-v_{\tau}$ symmetry limit, one can show that $Q_{21}=Q_{22}=Q_{23}=Q_{32}=0$. Therefore, under these two assumptions, there are only two free parameters, $Q_{31}$ and $Q_{33}$, for classifying all possible neutrino flavor transition models. This is a great advantage of $Q$ matrix parametrization for describing neutrino flavor transitions. The determinations of $Q_{31}$ and $Q_{33}$ by neutrino telescopes are discussed in Ref. [11].

\section{References}

[1] J. F. Beacom, N. F. Bell, D. Hooper, S. Pakvasa and T. J. Weiler, Phys. Rev. D 68, 093005 (2003) [Erratum-ibid. D 72, 019901 (2005)].

[2] E. Waxman and J. N. Bahcall, Phys. Rev. D 59, 023002 (1998).

[3] The subscript 0 is used to denote the neutrino flux at the source.

[4] K. C. Lai, G. L. Lin and T. C. Liu, Phys. Rev. D 80, 103005 (2009).

[5] For a review on neutrino flavor ratios in various astrophysical sources, see S. Pakvasa, Mod. Phys. Lett. A 19, 1163 (2004) [Yad. Fiz. 67, 1179 (2004)].

[6] M. C. Gonzalez-Garcia and M. Maltoni, Phys. Rept. 460, 1 (2008).

[7] T. C. Liu, M. A. Huang and G. .L. Lin, arXiv:1004.5154 [hep-ph].

[8] A. B. Balantekin and G. M. Fuller, Phys. Lett. B 471, 195 (1999).

[9] P. F. Harrison and W. G. Scott, Phys. Lett. B 547, 219 (2002).

[10] P. Allison et al., Nucl. Instrum. Meth. A 604, S64 (2009).

[11] K. C. Lai, G. L. Lin and T. C. Liu, Phys. Rev. D 82, 103003 (2010). 